\title{
Modern Approaches to Building Recommender Systems for Online Stores
}

\author{
Lyudmila Onokoy ${ }^{1}$, Jurijs Lavendels ${ }^{2 *}$ \\ ${ }^{1}$ Financial University under the Government of the Russian Federation, Moscow, Russia \\ ${ }^{2}$ Riga Technical University, Riga, Latvia
}

\begin{abstract}
The article presents current approaches to solving the problem of building recommender systems designed to intellectualize the user interface of online stores. Much attention is paid to modern methods of building recommender systems, analysing their strengths and weaknesses. Of greatest interest are the criteria for selecting effective methods for specific online stores and the authors' concept of a typical recommender system of electronic commerce.
\end{abstract}

Keywords - Collaborative filtering, combined co-filtering, content filtering, typical recommender system.

\section{INTRODUCTION}

Modern e-commerce is characterised by high development dynamics and broad coverage of the global economic space using the Internet. Sustainable annual growth of the sales volume in e-commerce [1] confirms the efficiency of this business area. According to eMarketer's retail, e-commerce sales are expected to reach $\$ 525.69$ bn. in 2018 [2]. However, the increase in online offers complicates the problem related to the search and choice of goods and services. This is determined by online availability of numerous offers similar by design and content in every category of goods and services, and this fact significantly complicates the search of the best online offers in view of buying preferences. As a result, duration of a real search often takes several hours.

To solve the problem of user interface intellectualization, online stores use recommender systems designed to ensure individualization and personalization of customer services.

It is known that recommender systems are designed to create recommendations for the buyers related to the choice of goods and services. These recommendations are created as a result of forecasting buying preferences based on the information already cumulated in a data system and considering buyer behaviours using the data mining and machine learning methods. Recommendations are published on a page of the website in the form of a small sample of goods offered for buying, usually under headings "Similar Goods" and/or "Frequently Bought Together". Today, recommender systems are actively used to form recommendations in such major online stores as Amazon, Ozon and others.

Currently, there are dozens of various recommender system building methods, and this factor complicates to a significant extent the problem of the most efficient choice between them in every particular case. The authors see the solution of this problem in elaboration of a typical recommender system designed for intellectualization of an online store's user interface. The functionality of this recommender system includes parametric tuning and adaptation to the specifics of a particular online store, the implementation of various forecasting methods, and the analysis of forecast accuracy.

\section{ClassifiCATION ANALYSIS OF MODERn APPROACHES TO BUILDING OF RECOMMENDER SYSTEMS}

Methods for constructing recommender systems [3], [4] are usually classified as follows (Fig. 1):

- collaborative filtering methods;

- content methods based on feature descriptions (contentbased, information filtering methods);

- methods based on knowledge of customer needs (knowledge-based methods);

- hybrid methods.

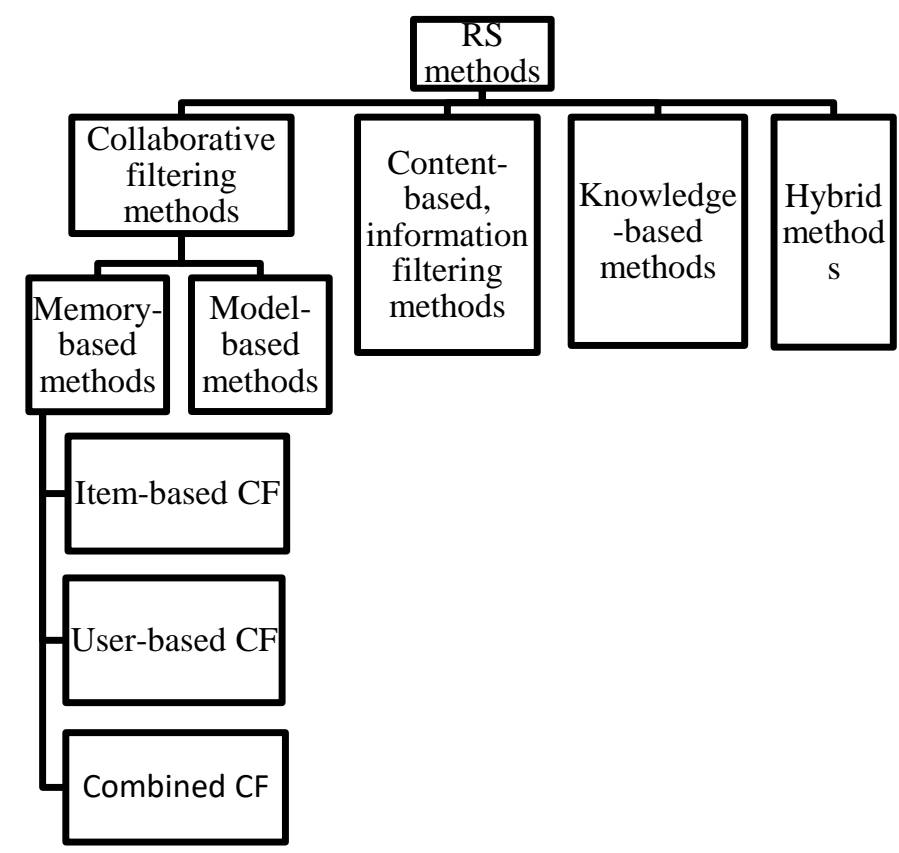

Fig. 1. Recommender system (RS) building methods.

* Corresponding author's e-mail: jurijs.lavendels@.rtu.lv 
Collaborative filtering methods [5] are based on the assumption that similar buyers make similar purchases, and shared goods are acquired by buyers together. The similarity of users in collaborative filtering is considered as the similarity of their behaviour, which is evaluated using explicit ratings (indicating the object's rating) and implicit ratings (recording the number of webpage views; estimating time spent on the webpage; registration of transition to another page with detailed information, etc.).

All collaborative-filtering methods are divided into two groups [6], [7]:

- anamnestic (memory-based) methods based on the analysis of all accumulated data;

- latent (model-based) methods based on the analysis of the data model.

In the methods based on the analysis of all accumulated data (memory-based) [8]-[11], the recommendations proposed to the buyer are formed using some measure calculated on the basis of all the accumulated data. These methods are divided into collaborative filtering by product similarity (item-based $\mathrm{CF}$ ) and user similarity (user-based CF), as well as a hybrid approach (combined collaborative filtering).

Filtering by products (item-based CF). In this case, the rating is assigned to the product on the basis of ratings of other similar products already included by the buyer in the order.

Item-based CF algorithm includes:

1. Development of a matrix of products to determine the degree of similarity between them.

2. Offering the products similar to those already ordered by the buyer based on a certain degree of similarity.

Let us view in detail the mathematical support of item-based $C F$. Let the data on $m$ products and $n$ transaction be available, then every $i$-transaction will be designated as $m$-dimensional vector:

$$
\begin{aligned}
& X_{i}=\left(x_{i, 1}, \ldots, x_{i, m}\right), \text { where } x_{i, j}, i \in\{1, \ldots n\} ; j \in\{1, \ldots, m\} \\
& x_{i, j} \text {-rating of } j \text {-product bought in } i \text {-transaction. }
\end{aligned}
$$

Every $j$-product will be designated as $n$-dimensional vector:

$$
Y_{j}=\left(x_{1, j}, \ldots, x_{n, j}\right) \text { rating of } j \text {-product in all transactions. }
$$

The item-based CF principle is to find the desired rating of the products in the transaction in question using the ratings of other products from this transaction. For example, you need to calculate the rating of item $i$, while in this transaction the rating of item $j$ is known. Then the rating $j$ will be used in the calculation of the rating $i$, in accordance with the frequency of occurrence of $i$ and $j$ in one transaction. Thus, the rating $i$ will be the higher, the higher in the analysed transaction the rating of other products, usually purchased together with the product $i$. Thus, the calculation of $\hat{x}_{u, j}$ - rating of the $j$-th product purchased in the $u$-th transaction will be carried out according to the formula:

$$
\hat{x}_{u, j}=\frac{\sum_{i=1}^{m} s_{i t e m s}\left(Y_{j}, Y_{i}\right) x_{u, i}}{\sum_{i=1}^{m}\left|S_{i t e m s}\left(Y_{j}, Y_{i}\right)\right|}
$$

where $S_{\text {items }}\left(Y_{j}, Y_{i}\right)$ is a measure of proximity of vectors of $i$ and $j$ products.

Sometimes in formula (1) instead of the value of the product rating $\left(x_{u, i}\right)$, the deviation of the rating $x_{u, i}$ from the average rating $i$ of the product for all transactions $\bar{y}_{i}$ is used:

$$
\hat{x}_{u, j}=\bar{y}_{j}+\frac{\sum_{i=1}^{m} s_{i t e m s}\left(Y_{j}, Y_{i}\right)\left(x_{u, i}-\bar{y}_{i}\right)}{\sum_{i=1}^{m}\left|S_{i t e m s}\left(Y_{j}, Y_{i}\right)\right|},
$$

where $\bar{y}_{i}=\frac{\sum_{k=1}^{n} x_{k, i}}{n}-$ average value of $i$-th item rating for all transactions.

Formulas (1), (2) have different representations, depending on the method used to calculate the proximity function $S_{i t e m s}\left(Y_{j}, Y_{i}\right)$ between the product rating vectors $i$ and $j-Y_{j}$ and $Y_{i}$.

Filtering by user (user-based CF). In this case, the product is assigned a rating, which is calculated using the ratings affixed to the same product by other buyers, but necessarily similar to this buyer.

User-based CF algorithm includes:

1. Search for the buyers similar to a given buyer (by certain characteristics taken from questionnaires or by similarity of transactions, i.e., similar purchases, for example).

2. Offering the products with the highest rating among the products that are chosen by similar buyers.

User-based CF uses practically the same mathematical methods that item-based CF. The only thing to be changed in the formulas describing these methods is the principle of the selection sequence of the $\mathrm{X}$ matrix elements.

The data to identify similarity of products, buyers and their transactions may be represented not only by ratings, but also by characteristics of products (colour, price, functionality etc.) and buyers (age, gender, information on buying preferences, level of income, etc.), transactions (behaviour of a buyer on the website).

Combined collaborative filtering methods [12] allow calculating the unknown rating as a result of using both two methods at once, namely buyer-based and item-based filtering. The required rating represents itself as a weighted sum of evaluations obtained as a result of applying these methods.

Another approach is to use mixed filtering based on similar product ratings in similar transactions. The decision in this case is made based on identification of a joint measure of similarity between the "transaction-product" pairs.

In the model-based methods analysing the formed models, recommendations offered to a buyer are formed based on the elaborated model of buying preferences, goods and interrelations between them. This group comprises the methods to be realised using one of the following approaches: Bayesian networks, cluster analysis, Markovian models, low-ranking matrix approximations [13]-[16]. 
Recommendation-forming algorithm with the use of modelbased CF methods consists of two stages:

1 learning a model in deferred mode;

2. development of solutions in real time based on the data of the trained model.

Content-based, information filtering methods [17]-[19] are based on the analysis of feature descriptions of goods and buyers. The key idea of content filtering is the assumption that the buyer is interested in the goods similar to those previously initiating his/her interest. Feature description-based approach assumes that there is a lot of knowledge related to the buyers and recommended goods. For example, all buyers fill the questionnaire where they register their social and demographic characteristics, interests etc. Sizes, colour, designation, price, brand etc. of the goods may be used as their descriptions in the online store. Based on the accumulated data on interactions between the buyers and goods, one may build the learning sample and narrow down the forecasted preference to a wellstudied task of precedent-based learning [16], [20].

Knowledge-based methods that use the knowledge of buyer's needs serve to build the expert recommended systems. Recommendations in the expert recommended systems are formed based on manually-formulated association rules. Such an approach may provide the very high quality of recommendations, though elaboration of such systems requires engagement of qualified experts to compile, update, review and add association rules.

Hybrid methods form the base for building the hybrid recommended systems. Hybrid recommended systems accumulate the advantages and compensate the weaknesses of various types of recommended systems. They are used to highlight seven different methods to build hybrid recommender systems.

1. Weighted methods. The resulting value of forecasted evaluation of a new product's buyer is calculated as a weighted arithmetic average of evaluations forecasted by various algorithms.

2. Switching methods. According to this approach, prior to form a recommendation, a value of some criterion is to be computed. Based on it, the decision is made about the choice of an algorithm to be applied to form recommendations. The criterion may represent itself as the comparison between the number of a user's actions and some preliminary chosen threshold, reaching of which may lead to the use of the CF method.

3. Combined methods. In this case, all recommendations are divided into $\mathrm{k}$ blocks, and recommendations for $j$ block are built using $j$-algorithm.

4. Feature-combining methods. According to this approach, features are obtained from various sources (e.g., social network data) and are further combined with already available features. According to practical experience, addition of new features improves the quality of recommendations.

5. Cascade methods. These methods anticipate iterative building of recommendations. The first-iteration algorithm plays the role of a rough filter, and further iterations improve its quality and adjust the obtained evaluations. Let the result of the first-iteration algorithm be the ranked list of objects V1. The subset of object U2 from the list of objects V1 with the highest forecasted evaluations enters the second iteration. After that, objects of subset U2 are ranked by the second-iteration algorithm. Traditionally, cascade methods use two iterations and two different algorithms, though, if necessary, the number of iterations (algorithms) may be increased.

6. Methods to increase the number of features. These methods are alike with the cascade ones. Their difference is that not the set of objects, but the set of tuples $<$ forecasted evaluation, object> is sent to the input of the second algorithm.

7. Meta-learning methods. According to this approach, the elaborated model learns based on the sample, features of which are the results of operation of different algorithms.

Hybrid methods may be divided into two categories, those are sequential and parallel ones. Parallel methods include weighted, combined, switching and feature-combined ones. Sequential methods include cascade, increasing number of features, and meta-learning.

According to the practical experience, all above-described methods have certain advantages and weaknesses that determine the choice of a method to build a particular recommended system.

\section{ADVANTAGES AND WEAKNESSES OF VARIOUS APPROACHES TO BUILDING OF RECOMMENDED SYSTEMS}

Based on the analysis of research results of other specialists [10], [11], [14], [15] and their own research, the authors formulated the main advantages and disadvantages of various methods of building recommender systems.

Advantages and weaknesses of memory-based CF methods:

- Advantages: simplicity, relatively high accuracy, easy data-adding algorithms - new transactions are added to the database and further be processed by the preset algorithms together with already existing data.

- Weaknesses: high time and memory expenditures; impossibility to generate forecasts for a new product or a user; impossibility of filtering, as the recommender system lacks the data on buying preferences ("cold start" problem) [15]; impossibility of the descriptive analysis of identified regularities and justification of forecasts; the problem of large dimensionality and sparseness (due to the lack of customer ratings for many products) of the rating matrix.

Advantages and weaknesses of model-based CF methods:

- Advantages: availability of a model ensuring the description and interpretation of the offered solutions.

- Weaknesses: typically, lower accuracy of the forecast compared with the memory-based approach; lack of simple data-adding algorithms, as addition of the new data to the database requires new calculation of the model. 
Advantages and weaknesses of content-based, information filtering CF methods:

- Advantages: the methods are devoid of the problems of collaborative filtering, such as the sparseness of the rating matrix, the cold start, since the methods are based on the analysis of the feature descriptions of goods and customers.

- Weaknesses: relatively low accuracy of forecasts (lower than that of collaborative filtering); big volume of input data; cold start of a user.

Advantages and weaknesses of knowledge-based methods:

- Advantages: interpretability of the compiled recommendations, easy realization based on the available set of association rules.

- Weaknesses: the complexity of the implementation in the absence of a set of associative rules and the difficulty of selecting experts.

Advantages of hybrid methods are determined by high accuracy of the forecasts, and the weaknesses - by highly complicated realization.

In practice, the choice of a certain method is determined by particular statement of a problem (specifics of a product range and buyers, technologies of storage and processing of information online store, etc.).

\section{SELECTION CRITERIA OF RECOMMENDER-BUILDING METHODS}

Theoretical studies performed by the authors, along with the practical experience, allowed shaping the criteria to select the methods of building recommender systems. Depending on the used method of building recommender systems, calculations and shaping of recommendations may be performed both in the delayed or online mode. That is why the choice of the type of recommendation system should consider the fact that the complex resource-intensive online calculations may significantly complicate operation of an online store and lead to substantially lower servicing of online buyers.

As for item-based CF methods, calculation of the extent of proximity of an analysed product and all other products may be performed in a delayed mode (typically during night, when the load on a website is minor), as the data on the goods are available till the decision-forming moment. Therefore, the entire algorithm of the work of the recommender system can be divided into two stages: the first stage (implemented in the deferred mode) is the calculation of the measure of proximity of goods to each other; the second stage (implemented in real time) is the calculation of product ratings. This approach can significantly reduce the resource intensity of the task of developing recommendations during the operation of the online store.

As for the user-based CF methods, the ability to calculate the extent of proximity of the buyers in the delayed mode depends on availability of the data on the analysed buyer in a recommended system, or this buyer is new. In the latter case, the formation of recommendations is possible only on the basis of the current transactions of the buyer and should occur in real time.
If transaction-based $\mathbf{C F}$ is applied, the measure of proximity between the analysed transaction and all other ones may be carried out online only, as the information on the current transaction will be available only at the moment of shaping of a recommendation.

Model-based methods are based on the concept of building of a model to form the solutions offered to buyers. In addition, models can be trained or configured using a database. To obtain the values of unknown ratings, as in the previously described methods, already established ratings are used, for which the classification function is calculated. This feature is subject to regular updates at the scheduled time to account for new transactions. Typically, the conversion of the classification function is produced during periods of the lowest load of computational tools, i.e., at night. Thus, the decision-making process in the model-based methods consists of two stages, those are delayed periodical learning and online calculation of estimated rank values. If the first stage implements the resource-intensive calculation, along with preservation of the parameter values and the structure of a model, the second stage is characterised by relatively low resource consumption. Therefore, the advantage of model-based methods lays in optimization of operation performance time.

Content-based, information filtering methods may be implemented in a deferred mode only to assess the similarity of goods. Evaluation of the similarity of buyers requires the analysis of their current behaviour, which is implemented in real time.

Allowable calculation modes in expert and hybrid recommender systems depend on the applied mathematical methods and requirements for the immediacy of the initial data.

If the range of a web-store goods anticipates non-frequent renewal of the line of goods, and one should acquire many goods with the others that add to their functions or are used during their installation (adjustment), we may recommend item-based CF as a method to build the recommender system.

If an online store sells specific goods (e.g., the tools and spare parts for a particular activity) of interest for a particular target buying audience, and new buyers are rare, then user-based CF may be recommended as a method to build the recommender system.

If there is a lot of information on the buyers and recommended goods of an online store, or such information can be obtained in sufficient volumes, the content-based, information filtering methods may be recommended.

Expert recommender systems have proved their best for online stores selling the unique rarely purchased goods, such as houses, cars or expensive items of luxury. In these cases, the recommendation-shaping process is complicated by the lack of rankings of the goods. Recommendations in the expert systems are offered not based on the rankings, but rather on similarity between user requirements and descriptions of the goods, or depending on the limitations specified by a user during specialization of a desired product. This type of a recommender system allows the client to specify in detail what he wants. 
If the accuracy of a forecast is a critical requirement to the recommender system, then the hybrid approach may be used for its building.

Reliability of forecasts obtained with the use of a chosen method is determined empirically.

\section{CONCEPT OF THE E-COMMERCE RECOMMENDER SYSTEM}

Currently, large online stores use unique recommendation systems, developed taking into account their specifics. In turn, smaller online stores are interested as well in enhancing the quality of servicing their web clients. This problem, according to the authors, can be solved by a typical recommender system that allows predicting the behaviour of customers using fairly simple and well-proven methods of collaborative and/or content filtering. It is known that a typical information system is designed for automation of a standard set of functionalities of similar organisations. The key advantage of the typical recommender system offered by the authors is that it can be customized for a particular online store, up to the selection of a particular building method.

In view of the above said, a typical recommender system for online stores consists of the following subsystems (Fig. 2):

- customization and adaptation to customer requirements;

- gathering of data;

- processing of buyer transactions;

- shaping of recommendations;

- analysis of the reliability of forecasts and self-learning of the system.

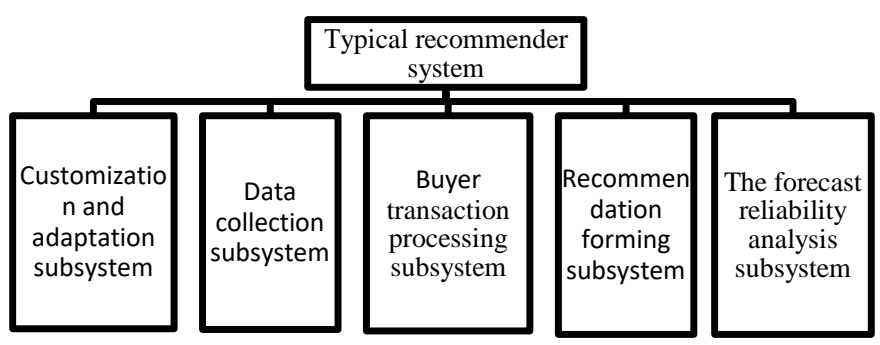

Fig. 2. Structure of a typical recommender system for online stores.

The customization and adaptation subsystem to customer requirements is designed for parametric and algorithmic adjustment of the system to the specifics of a particular online store according to the following indicators:

- Characteristics taken into account when determining the similarity of goods (for example, price, size, technical parameters, colour, etc.) and their weight parameters;

- Characteristics taken into account in determining the similarity of customers (for example, age, gender, financial status, marital status, etc.) and their weight parameters;

- Characteristics taken into account when determining the similarity of customer behaviour (for example, ratings, purchased goods, etc.);
- Algorithm for shaping association rules connecting the registered stable buying user preferences and recommendations of the system;

- Customer Questionnaire, which includes both general questions for registering such customer parameters as gender, age, etc., and questions about customer preferences;

- Parameters of buyer activity logging registered in the system and algorithms of their accounting in the rating evaluation of goods by buyers.

In addition, the initial system's setting allows for selection of recommendation-shaping modules (methods of building recommender system) and algorithms of their use. As a result, from the selected modules of recommendations, a recommendation system is designed that meets the specifics of a particular online store in terms of the range of goods, target groups of buyers and their behaviour.

The statistical data accumulated by the online store, the customer database of the CRM system and/or expert evaluations of the online store managers can be used as the initial data for initial parametric setting.

During operation of the system, the administrator may introduce the changes into parameter settings based on the results of the analysis of the performance efficiency indicators of the recommender system that are formed as output data of the forecast reliability analysis subsystem.

The data collection subsystem implements the following data accumulation types:

Explicit collection of the data on characteristics of the goods, etc.

Explicit collection of the data on characteristics of the buyers, exposed ratings, filled questionnaires.

The buyer transaction processing subsystem is designed for non-explicit data collection based on logging of a buyer's behaviour and creation of a good's ranking based on the results of analysis and processing of a buyer's behaviour in a given transaction, as the explicit assignation of a good's rating by the buyer is not always possible.

This subsystem considers the following behaviour characteristics of buyers:

- Fact of buying a product (determines the high rating of this product for a buyer);

- Amount of the goods bought;

- Viewing information about the product for a period of time longer than the specified limit number set in the system setup mode;

- Switch to a webpage with detailed information on the product;

- Number of a repeated access to the webpage with the information on this product.

The recommendation forming subsystem is based on the collaborative and content filtering methods chosen at the system setting stage. This subsystem consists of the item- and/or userbased CF modules, combined joint filtering, content filtering by goods and buyers.

In addition, the recommendation shaping subsystem includes the module of creating recommendations based on sustainable 
preferences of a buyer. If the buyer has repeatedly accessed the site, and his purchases are registered in the database, then the system forms recommendations with regard to his/her particular buying preferences, using association rules logged at the system setting stage.

For example, the association rule-shaping principle may be as follows: If Product 1 is chosen, then, typically, this buyer purchases Product 2 with it, so, Product 2 will be recommended for the buyer for purchasing. If the choice of the first product for this buyer has analogues in previous transactions, then the goods to be recommended are most likely chosen among those that were included into the previous transactions together with the chosen product.

In every particular case of introduction of a typical recommender system, the choice of recommendation shaping methods is determined by a web-store specifics.

The forecast reliability analysis subsystem is designed for collection of the statistical data on compliance of recommended goods with the actual choice of a buyer. The most widely spread metrics for assessing the quality of recommender systems include precision and recall of the forecast. Precision is determined as a share of objects interesting for a buyer among the offered recommendations. Recall is determined as a share of recommended objects among all objects interesting for a buyer [6].

The recommender system includes the self-learning mode for selecting the most productive methods to shape the recommendations by the system. In case of a high share percentage of discrepancies between the recommended and the chosen goods, re-setting of the system and the choice of other methods is possible.

The authors' concept of the model recommendation system described above has become the basis for its further development.

\section{CONCLUSION}

The active development of modern e-commerce is determined partly by introduction of new methods and processes of intellectual data analysis, including recommendation systems. According to the practical experience, the recommender system facilitates the enhancement of e-commerce quality and efficiency, namely:

- reduction of labour expenditures of consulting managers who, answering the phone calls from buyers, have to compensate the lack of data recall and precision in the description of goods and services on the webpages of an online store;

- providing customers with the opportunity to move through the site on their own adapted trajectory;

- implementation of a differential approach to creation of content for webpages of an online store, which meets individual preferences of a buyer;

- enhancement of immediacy and productivity of a buyer's search for a product or a service.

The concept of a typical recommender system developed by the authors is based on the use of the proven collaborative and content filtering methods. Its key advantages include broad capabilities related to adjustment and customization of the system with regard to a particular online store specifics; solution of the "cold start" problem using content-filtering methods, selflearning of the system and selection of the most efficient mathematical methods.

The concept of a typical recommender system offered by the authors is the next contribution to advancement of the ideas related to intellectualization of informational support to ecommerce, and it will be interesting for developers of web applications and recommender systems, as well as other online business experts.

\section{REFERENCES}

[1] E-commerce trends. [Online] Available from: https://don16obqbay2c.cloudfront.net/wp-content/uploads/ru/Retina_Ecommerce-trends_Part2-1480613113.png

[2] eMarketer. Top 10 US Ecommerce Companies in 2018. [Online] Available from: https://www.emarketer.com/content/top-10-usecommerce-companies-in-2018

[3] G. Adomavicius and A. Tuzhilin, "Toward the next generation of recommender systems: A survey of the state-of-the-art and possible extensions," IEEE Transactions on Knowledge and Data Engineering, vol. 17, no. 6, pp. 734-749, 2005. https://doi.org/10.1109/TKDE.2005.99

[4] A. G. Gomzin, A. V. Korshunov, "Recommender systems: a survey of modern approaches," Proceedings of the Institute for System Programming, vol. 22, 2012, pp. 401-418. https://doi.org/10.15514/ISPRAS-2012-22-21

[5] F. M. Harper and J. A. Konstan, "The MovieLens Datasets: History and Context," ACM Transactions on Interactive Intelligent Systems (TiiS), vol. 5, no. 4, 2016. https://doi.org/10.1145/2827872

[6] G. Shani and A. Gunawardana, "Evaluating recommendation systems", Recommender systems handbook. Springer, 2011. pp. 257-297. https://doi.org/10.1007/978-0-387-85820-3 8

[7] X. Su and T. M. Khoshgoftaar, "Survey of collaborative Filtering Techniques," Advances in Artifical Intelligence, vol. 2009. https://doi.org/10.1155/2009/421425

[8] Y. Koren, R. Bell, and C. Volinsky, "Matrix factorization techniques for recommender systems," Computer, vol. 42, no. 8, pp. 30-37, 2009. https://doi.org/10.1109/MC.2009.263

[9] D. Lemire and A. Maclachlan, "Slope one predictors for online ratingbased collaborative filtering," Proceedings of the 2005 SIAM International Conference on Data Mining, pp. 471-475, 2005. https://doi.org/10.1137/1.9781611972757.43

[10] J. Wang, A. P. de Vries, and M. J. T. Reinders, "Unifying user-based and item-based collaborative filtering approaches by similarity fusion. In Proceedings of the 29th annual international ACM SIGIR conference on Research and development in information retrieval, pp. 501-508, 2006. https://doi.org/10.1145/1148170.1148257

[11] C. Desrosiers and G. Karypis, A comprehensive survey of neighborhoodbased recommendation methods. Recommender systems handbook, F. Ricci et al. (eds), pp. 107-144. Springer, 2011. https://doi.org/10.1007/978-0-387-85820-3_4

[12] Y. Koren, "Factorization meets the neighborhood: a multifaceted collaborative filtering model," in Proceedings of the 14th ACM SIGKDD international conference on Knowledge discovery and data mining, pp. 426-434, ACM, 2008. https://doi.org/10.1145/1401890.1401944

[13] A. Hernando, J. Bobadilla, and F. Ortega, "A non negative matrix factorization for collaborative filtering recommender systems based on a Bayesian probabilistic model," Knowledge-Based Systems, vol. 97, pp. 188-202, 2016. https://doi.org/10.1016/j.knosys.2015.12.018

[14] H. Steck, "Training and testing of recommender systems on data missing not at random," in Proceedings of the 16th ACM SIGKDD international conference on Knowledge discovery and data mining, pp. 713-722, ACM, 2010. https://doi.org/10.1145/1835804.1835895

[15] A. I. Schein, A. Popescul, L. H. Ungar, and D. M. Pennock, "Methods and metrics for cold-start recommendations," in Proceedings of the 25th annual international ACM SIGIR conference on Research and development in information retrieval, pp. 253-260, ACM, 2002. https://doi.org/10.1145/564418.564421 
[16] S. Rendle, "Factorization machines," in Proceedings of the 10th IEEE International Conference on Data Mining, IEEE, 2010. https://doi.org/10.1109/ICDM.2010.127

[17] Jannach D., Zanker M., Felfernig A., Friedrich G. Recommender Systems. An Introduction. New York: Cambridge University Press, 2011, $352 \mathrm{p}$.

[18] P. Melville and V. Sindhwani, "Recommender Systems," In: C. Sammut and G. Webb, Eds., Encyclopedia of Machine Learning, Springer, Berlin, 2010, pp. 829-838

[19] M. Tim Jones, "Ai Application Programming (Programming Series)", 2nd edition, Charles River Media, 2005.

[20] L. Wang, L. Cheng, and G. Zhao, "Machine Learning for Human Motion Analysis: Theory and Practice," IGI Global, 2009. https://doi.org/10.4018/978-1-60566-900-7

[21] R. Burke, "Hybrid web recommender systems," The adaptive web, Lecture Notes in Computer Science, Springer, pp. 377-408, 2007. https://doi.org/10.1007/978-3-540-72079-9_12

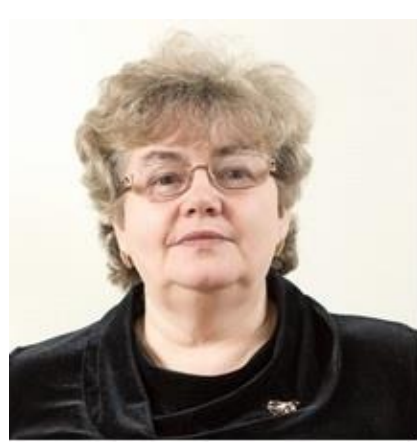

Lyudmila Onokoy, Doctor of sociology. She is a Professor of Business Informatics Department at the Financial University under the Government of the Russian Federation (Moscow). Her scientific and pedagogical work experience is 39 years.

The main area of research is development of techniques and methodologies of software engineering. Phone: +9154863392

E-mail: OnokoyLS@fa.ru

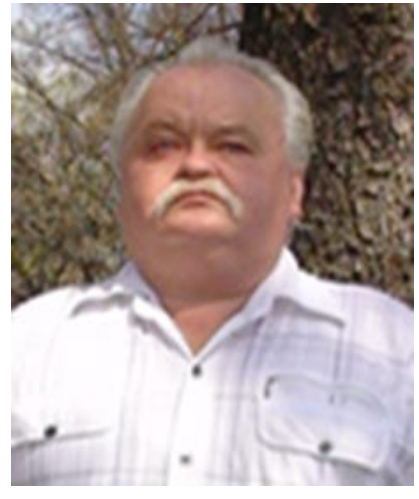

Jurijs Lavendels, Dr. sc. ing. He is a Professor at Riga Technical University. His current research interests include computation methods with discretisation and algebraisation for design and modelling of physical processes.

Address: Kalku Street 1, Riga, LV-1658, Latvia

Phone +371 67089573

E-mail: jurijs.lavendels@.rtu.lv

ORCID iD: https://orcid.org/00000002-8448-4705 